\title{
On the Solution of Korteweg de Vries Equation by Laplace Homotopy Pertubation Method
}

\author{
Wanchak Satsanit $^{1} \&$ Kankullanat Arnuphap ${ }^{1}$ \\ ${ }^{1}$ Department of Mathematics, Faculty of Science, Maejo University Chiangmai, 50290, Thailand \\ Correspondence: Wanchak Satsanit, Department of Mathematics, Faculty of Science, Maejo University Chiangmai, \\ 50290, Thailand. E-mail: wanchack@gmail.com
}

Received: March 4, 2019 Accepted: July 26, 2019 Online Published: July 30, 2019

doi:10.5539/jmr.v11n4p77

URL: https://doi.org/10.5539/jmr.v11n4p77

\begin{abstract}
In this paper, we applied Laplace Homotopy Pertubation method (LHPM) for solving Korteveg de Vries equation. It was found that this method yields a very rapid convergence of the solution series which leads the solution in a closed form.
\end{abstract}

Keywords: Korteveg de Vries equation, Laplace transform, Homotopy pertubation method

Mathematics Subject Classification: 46F10, 46F12.

\section{Introduction}

It is well known that the linear and nonlinear partial differential equation are widely used to describe in variety fields os science such as physics, biology, chemistry, etc. There are several transform to solve partial differential equation such as Laplace transform, Fourier transform, Wavelet transform etc.Homotopy Pertubation method (HPM) first introduced by Ji-Huen He (1990 \& 2016) for solving differential and integral equations, linear and nonlinear partial differential equation. The HPM yields a very rapid convergence of the solution series in most cases. This He's HPM is a method which can be solve various kinds of linear and nonlinear partial differential equation. In 2007, Monami and Odibat (2007 \& 2005) applied homotopy pertubation method (HPM) to fractional differential equation and showed that HPM is an alternative analytical method for fractional differential equation.Furthermore,J. Singh, D.Kumar[3]have studied Homotopy Pertubation Transform Method for solving Fractional Heat and Wave-Like Equations.

The purpose of this work, Korteweg de Vries as follows

$$
u_{t}+a u u_{x}+u_{x x x}=0, a \in R
$$

with the initial condition

$$
u(x, 0)=f(x)
$$

By homotopy pertubation method combined Laplace transform, we obtain the solution in the infinite series form. Severals examples are given to verify the reliability and efficiency of this method. Before going to that point, the following definition and some important concepts are needed.

\section{Preliminaries}

In this section, we are giving some basic definition and proportion which shall be in used in this paper:

Definition 1 The Laplace transform of a functional $f(t), t>0$ is defined as

$$
\mathcal{L}[f(t)]=F(s)=\int_{0}^{\infty} e^{-s t} f(t) d t,
$$

where $f(t)$ is piecewise continuous and of the exponential order (i.e. $\left|e^{-a t} f(t)\right|<M$ ) for some constants $a$, $M$ and complex parameter $s$. 
Definition 2 The Laplace transform of the derivative is given by the following form

$$
\mathcal{L}[D f(t)]=s^{n} \mathcal{L}[f(t)]-\sum_{r=0}^{n-1} s^{n-r-1} f^{(r)}(0+)
$$

\section{Homotopy Perturbation Method}

Consider the following general nonlinear differential equation:

$$
L u+N u=g(x, t),
$$

with initial conditions

$$
u(x, 0)=k_{1}, u_{t}(x, 0)=k_{1}
$$

where $u$ is a function of $x$ and $t$ and $c_{1}, c_{2}$ are constants or functions of $x$, and $L$ and $N$ are the linear and nonlinear operators respectively.

According to HPM, we construct a homotopy which satisfies the following relation

$$
H(u, p)=(1-p)\left[L u-L u_{0}\right]+p[L u+N u-g(x, t)]=0
$$

where $p \in[0,1]$ is an embedding parameter and $u_{0}$ is an arbitrary initial approximation satisfing the given initial conditions. When we put $p=0$ and $p=1$ in Eq. (2.5), we obtain

$$
H(u, 0)=L u-L u_{0}=0
$$

and

$$
H(u, 1)=L u+N u-g(x, t)=0
$$

In HPM, the solution of Eq. (2.5) is expressed as

$$
u(x, t)=u_{0}(x, t)+p u_{1}(x, t)+p^{2} u_{2}(x, t)+\ldots
$$

Hence, the approximate solution of Eq. (2.3) can be expressed as a series of the powers of $p$, i.e.

$$
\begin{aligned}
u(x, t) & =\lim _{p \rightarrow 1}\left(u_{0}(x, t)+p u_{1}(x, t)+p^{2} u_{2}(x, t)+\ldots\right) \\
& =u_{0}(x, t)+u_{1}(x, t)+u_{2}(x, t)+\ldots
\end{aligned}
$$

\section{Laplace Homotopy Perturbation Method (LHPM)}

To illustrate the basic idea of this method, we consider a general nonlinear nonhomogeneous partial differential equation with the initial conditions of the form:

$$
\begin{array}{r}
D_{t} u(x, t)+R u(x, t)+N u(x, t)=g u(x, t), \\
u(x, 0)=h(x), u_{t}(x, 0)=f(x),
\end{array}
$$

where $D_{t} u(x, t)$ is the second order linear differential equation, $R$ is the linear differential operator of less order than $D, N$ represents the general nonlinear differential operator and $g(x, t)$ is the source term. Taking the Laplace transform (denoted in this paper by $L$ ) on both sides of (2.11), we get

$$
\mathcal{L}\left[D_{t} u(x, t)\right]+\mathcal{L}[R u(x, t)]+\mathcal{L}[N u(x, t)]=\mathcal{L}[g u(x, t)]
$$

Using the property of the Laplace transform, we have

$$
\mathcal{L}[u(x, t)]=\frac{h(x)}{s}+\frac{f(x)}{s^{2}}+\frac{1}{s^{n}} \mathcal{L}[g u(x, t)]-\frac{1}{s^{n}} \mathcal{L}[R u(x, t)]-\frac{1}{s^{n}} \mathcal{L}[N u(x, t)]
$$


Operating with the Laplace inverse on both sides of (2.11), we get

$$
u(x, t)=G(x, t)-\mathcal{L}^{-1}\left[\frac{1}{s^{n}} \mathcal{L}[R u(x, t)+N u(x, t)],\right.
$$

where $G(x, t)$ represents the term arising form source term and the prescribed initial condition. Now we apply the LHPM

$$
u(x, t)=\sum_{n=0}^{\infty} p^{n} u_{n}(x, t)
$$

and the nonlinear term can be decomposed as

$$
N(u)=\sum_{n=0}^{\infty} p^{n} H_{n}(u)
$$

for some He's polynomials $H_{n}(u)$ that are given by

$$
H_{n}\left(u_{0}, u_{1}, \ldots, u_{n}\right)=\frac{1}{n !} \frac{d^{n}}{d p^{n}}\left[N\left(\sum_{i=0}^{\infty} p^{i} u_{i}(x, t)\right)\right]_{p=0} ; n=0,1,2, \ldots
$$

The first few components He's are given by

$$
\begin{aligned}
& H_{0}=N\left(u_{0}\right) \\
& H_{1}=u_{1} N^{\prime}\left(u_{0}\right) \\
& H_{2}=u_{2} N^{\prime}\left(u_{0}\right)+\frac{1}{2} u_{1}^{2} N^{\prime \prime}\left(u_{0}\right) \\
& H_{3}=u_{3} N^{\prime}\left(u_{0}\right)+u_{1} u_{2} N^{\prime \prime}\left(u_{0}\right) \frac{u_{1}^{3}}{3 !} N^{(3)}\left(u_{0}\right)
\end{aligned}
$$

We apply homotopy pertubation method in (2.15), we obtain

$$
\sum_{n=0}^{\infty} p^{n} u_{n}(x, t)=G(x, t)-p\left(\mathcal{L}^{-1}\left[\frac{1}{s^{n}} \mathcal{L}\left[R \sum_{n=0}^{\infty} p^{n} u_{n}(x, t)+N \sum_{n=0}^{\infty} p^{n} u_{n}(x, t)\right]\right]\right) .
$$

We compare the coefficients of like powers of $p$, the following approximations are obtained.

$$
\begin{aligned}
& p^{0}: u_{0}(x, t)=G(x, t) \\
& p^{1}: u_{1}(x, t)=-\mathcal{L}^{-1}\left[\frac{1}{s^{n}} \mathcal{L}\left[R u_{0}(x, t)-H_{0}(u)\right]\right] \\
& p^{2}: u_{2}(x, t)=-\mathcal{L}^{-1}\left[\frac{1}{s^{n}} \mathcal{L}\left[R u_{1}(x, t)-H_{1}(u)\right]\right] \\
& p^{3}: u_{3}(x, t)=-\mathcal{L}^{-1}\left[\frac{1}{s^{n}} \mathcal{L}\left[R u_{2}(x, t)-H_{2}(u)\right]\right]
\end{aligned}
$$

Finally, we approximate the analytical solution $u(x, t)$ by truncated series

$$
u(x, t)=\lim _{N \rightarrow \infty} \sum_{n=0}^{N} u_{n}(x, t)
$$

\section{Examples}

To illustrate the basic idea of this method, we consider a general Korteweg De Vries(KDV) equation with the initial conditions. 
Example 1 Consider the following one-dimension linear KDV equation.

$$
u_{t}+u_{x x x}=0
$$

with the initial condition $u(x, 0)=e^{x}$.

Taking the Laplace transform on both sides of Eq.(3.1), we get

$$
\begin{array}{r}
\mathcal{L}\left[u_{t}\right]+\mathcal{L}\left[u_{x x x}\right]=\mathcal{L}[0] \\
s u(x, s)-u(x, 0)+\mathcal{L}\left[u_{x x x}\right]=0 \\
s u(x, s)=e^{x}-\mathcal{L}\left[u_{x x x}\right] \\
u(x, s)=\frac{e^{x}}{s}-\frac{1}{s} \mathcal{L}\left[u_{x x x}\right] \\
u(x, t)=e^{x}-\mathcal{L}^{-1}\left[\frac{1}{s} \mathcal{L}\left[u_{x x x}\right]\right]
\end{array}
$$

Now,we apply the homotopy perturbation method,we get

$$
\sum_{n=0}^{\infty} p^{n} u_{n}(x, t)=e^{x}-p\left(\mathcal{L}^{-1}\left[\frac{1}{s} \mathcal{L}\left[\left(\sum_{n=0}^{\infty} p^{n} u_{n}(x, t)\right)_{x x x}\right]\right]\right)
$$

$$
p^{0} u_{0}(x, t)+p^{1} u_{1}(x, t)+p^{2} u_{2}(x, t)+\ldots
$$

$$
=e^{x}-p\left(\mathcal{L}^{-1}\left[\frac{1}{S} \mathcal{L}\left[\left(p^{0} u_{0}(x, t)+p^{1} u_{1}(x, t)+\ldots\right)_{x x x}\right]\right]\right)
$$

Comparing the coefficient of like power of $p$, the following approximations are obtained,

$$
\begin{aligned}
& p^{0}: u_{0}(x, t)=e^{x} \\
& p^{1}: u_{1}(x, t)=-\mathcal{L}^{-1}\left[\frac{1}{s} \mathcal{L}\left[\left(u_{0}(x, t)\right)_{x x x}\right]\right] \\
&=-\mathcal{L}^{-1}\left[\frac{1}{s} \mathcal{L}\left[\left(e^{x}\right)_{x x x}\right]\right] \\
&=-e^{x} \mathcal{L}^{-1}\left[\frac{1}{s^{2}}\right] \\
&=-e^{x} t \\
& p^{2}: u_{2}(x, t)=-\mathcal{L}^{-1}\left[\frac{1}{s} \mathcal{L}\left[\left(u_{1}(x, t)\right)_{x x x}\right]\right] \\
&=-\mathcal{L}^{-1}\left[\frac{1}{s} \mathcal{L}\left[\left(-e^{x} t\right)_{x x x}\right]\right] \\
&=e^{x} \frac{t^{2}}{2 !} \\
& p^{3}: u_{3}(x, t)=-\mathcal{L}^{-1}\left[\frac{1}{s} \mathcal{L}\left[\left(u_{2}(x, t)\right)_{x x x}\right]\right] \\
&=-\mathcal{L}^{-1}\left[\frac{1}{s} \mathcal{L}\left[\left(e^{x} \frac{t^{2}}{2 !}\right)_{x x x}\right]\right] \\
&=e^{x} \frac{t^{4}}{4 !} \\
&=-e^{x} \frac{t^{3}}{3 !} \\
& p^{4}: u_{4}(x, t)=-\mathcal{L}^{-1}\left[\frac{1}{s} \mathcal{L}\left[\left(u_{3}(x, t)\right)_{x x x}\right]\right] \\
&\left.\mathcal{L}\left[\left(-e^{x} \frac{t^{3}}{3 !}\right)_{x x x}\right]\right] \\
& \\
&
\end{aligned}
$$


Therefore the solution $u(x, t)$ is given by :

$$
\begin{aligned}
u(x, t) & =u_{0}(x, t)+u_{1}(x, t)+u_{2}(x, t)+u_{3}(x, t)+\ldots \\
& =e^{x}-e^{x} t+e^{x} \frac{t^{2}}{2 !}-e^{x} \frac{t^{3}}{3 !}+e^{x} \frac{t^{4}}{4 !}+\ldots \\
& =e^{x}\left(1-t+\frac{t^{2}}{2 !}-\frac{t^{3}}{3 !}+\frac{t^{4}}{4 !}+\ldots\right) \\
& =e^{x-t}
\end{aligned}
$$

Example 2 Consider the following two-dimensions linear KDV equation.

$$
u_{t}+u_{x x x}+u_{y y y}=0
$$

with the initial condition $u(x, y, 0)=e^{x+y}$

Taking the Laplace transform on both sides of Eq. (3.3),we get

$$
\begin{array}{r}
\mathcal{L}\left[u_{t}\right]+\mathcal{L}\left[u_{x x x}\right]+\mathcal{L}\left[u_{y y y}\right]=\mathcal{L}[0] \\
s u(x, y, s)-u(x, y, 0)+\mathcal{L}\left[u_{x x x}\right]+\mathcal{L}\left[u_{y y y}\right]=0 \\
\operatorname{su}(x, y, s)=e^{x+y}-\mathcal{L}\left[u_{x x x}\right]-\mathcal{L}\left[u_{y y y}\right]=0 \\
u(x, y, s)=\frac{e^{x+y}}{s}-\frac{1}{s} \mathcal{L}\left[u_{x x x}+u_{y y y}\right]
\end{array}
$$

The inverse of Laplace transform implies that

$$
\begin{aligned}
u(x, y, t) & =\mathcal{L}^{-1}\left[\frac{e^{x+y}}{s}\right]-\mathcal{L}^{-1}\left[\frac{1}{s} \mathcal{L}\left[u_{x x x}+u_{y y y}\right]\right] \\
& =e^{x+y} \mathcal{L}^{-1}\left[\frac{1}{s}\right]-\mathcal{L}^{-1}\left[\frac{1}{s} \mathcal{L}\left[u_{x x x}+u_{y y y}\right]\right] \\
& =e^{x+y}-\mathcal{L}^{-1}\left[\frac{1}{s} \mathcal{L}\left[u_{x x x}+u_{y y y}\right]\right]
\end{aligned}
$$

Now,we apply the homotopy perturbation method, we get

$$
\sum_{n=0}^{\infty} p^{n} u_{n}(x, y, t)=e^{x+y}-p\left(\mathcal{L}^{-1}\left[\frac{1}{s} \mathcal{L}\left[\left(\sum_{n=0}^{\infty} p^{n} u_{n}(x, y, t)\right)_{x x x}+\left(\sum_{n=0}^{\infty} p^{n} u_{n}(x, y, t)\right)_{y y y}\right]\right]\right)
$$

$$
\begin{aligned}
& p^{0} u_{0}(x, y, t)+p^{1} u_{1}(x, y, t)+p^{2} u_{2}(x, y, t)+\ldots= \\
& e^{x+y}-p\left(\mathcal { L } ^ { - 1 } \left[\frac { 1 } { s } \mathcal { L } \left[\left(p^{0} u_{0}(x, y, t)+p^{1} u_{1}(x, y, t)+\ldots\right)_{x x x}\right.\right.\right. \\
& \left.\left.\left.+\left(p^{0} u_{0}(x, y, t)+p^{1} u_{1}(x, y, t)+\ldots\right)_{y y y}\right]\right]\right)
\end{aligned}
$$

Comparing the coefficient of like power of $p$, the following approximations are 
obtained;

$$
\begin{aligned}
p^{0}: u_{0}(x, y, t) & =e^{x+y} \\
p^{1}: u_{1}(x, y, t) & =-\mathcal{L}^{-1}\left[\frac{1}{s} \mathcal{L}\left[\left(u_{0}(x, y, t)\right)_{x x x}+\left(u_{0}(x, y, t)\right)_{y y y}\right]\right] \\
& =-\mathcal{L}^{-1}\left[\frac{1}{s} \mathcal{L}\left[\left(e^{x+y}\right)_{x x x}+\left(e^{x+y}\right)_{y y y}\right]\right] \\
& =-2 e^{x+y} t \\
p^{2}: u_{2}(x, y, t) & =-\mathcal{L}^{-1}\left[\frac{1}{s} \mathcal{L}\left[\left(u_{1}(x, y, t)\right)_{x x x}+\left(u_{1}(x, y, t)\right)_{y y y}\right]\right] \\
& =-\mathcal{L}^{-1}\left[\frac{1}{s} \mathcal{L}\left[\left(-2 e^{x+y} t\right)_{x x x}+\left(-2 e^{x+y} t\right)_{y y y}\right]\right] \\
& =2 e^{x+y} t^{2} \\
p^{3}: u_{3}(x, y, t) & =-\mathcal{L}^{-1}\left[\frac{1}{s} \mathcal{L}\left[\left(u_{2}(x, y, t)\right)_{x x x}+\left(u_{2}(x, y, t)\right)_{y y y}\right]\right] \\
& =-\mathcal{L}^{-1}\left[\frac{1}{s} \mathcal{L}\left[\left(2 e^{x+y} t^{2}\right)_{x x x}+\left(2 e^{x+y} t^{2}\right)_{y y y}\right]\right] \\
& =-4 e^{x+y} \frac{t^{3}}{3}
\end{aligned}
$$

Therefore the solution $u(x, t)$ is given by :

$$
\begin{aligned}
u(x, y, t) & =u_{0}(x, y, t)+u_{1}(x, y, t)+u_{2}(x, y, t)+u_{3}(x, y, t)+\ldots \\
& =e^{x+y}-2 e^{x+y} t+2 e^{x+y} t^{2}-4 e^{x+y} \frac{t^{3}}{3}+\ldots \\
& =e^{x+y}\left(1-2 t+\frac{4 t^{2}}{2 !}-\frac{8 t^{3}}{3 !}+\ldots\right) \\
& =e^{x+y-2 t}
\end{aligned}
$$

Example 3 Consider the following three-dimensions inhomogeneous linear KDV-like equation.

$$
\frac{\partial u}{\partial t}+\frac{1}{72}\left(x^{3} u_{x x x}+y^{3} u_{y y y}+z^{3} u_{z z z}\right)=x^{4} y^{4} z^{4}
$$

with the initial condition $u(x, y, z, 0)=0$.

Taking the Laplace transform on both sides of Eq. (3.9),we get

$$
\begin{array}{r}
\mathcal{L}\left[u_{t}\right]+\frac{1}{72} \mathcal{L}\left[x^{3} u_{x x x}+y^{3} u_{y y y}+z^{3} u_{z z z}\right]=\mathcal{L}\left[x^{4} y^{4} z^{4}\right] \\
s u(x, y, z, s)-u(x, y, z, 0)+\frac{1}{72} \mathcal{L}\left[x^{3} u_{x x x}+y^{3} u_{y y y}+z^{3} u_{z z z}\right]=\frac{x^{4} y^{4} z^{4}}{s} \\
u(x, y, z, s)=-\frac{1}{72 s} \mathcal{L}\left[x^{3} u_{x x x}+y^{3} u_{y y y}+z^{3} u_{z z z}\right]+\frac{x^{4} y^{4} z^{4}}{s^{2}}
\end{array}
$$

Taking inverse Laplace transform both sides of the above equation, we obtain

$$
\begin{aligned}
u(x, y, z, t) & =-\mathcal{L}^{-1}\left[\frac{1}{72 s} \mathcal{L}\left[x^{3} u_{x x x}+y^{3} u_{y y y}+z^{3} u_{z z z}\right]\right]+\mathcal{L}^{-1}\left[\frac{x^{4} y^{4} z^{4}}{s^{2}}\right] \\
& =x^{4} y^{4} z^{4} t-\mathcal{L}^{-1}\left[\frac{1}{72 s} \mathcal{L}\left[x^{3} u_{x x x}+y^{3} u_{y y y}+z^{3} u_{z z z}\right]\right]
\end{aligned}
$$

Now,we apply the homotopy perturbation method, we get

$$
\begin{aligned}
\sum_{n=0}^{\infty} p^{n} u_{n}(x, y, z, t)=x^{4} y^{4} z^{4} t-p\left(\mathcal { L } ^ { - 1 } \left[\frac { 1 } { 7 2 s } \mathcal { L } \left[x^{3}\left(\sum_{n=0}^{\infty} p^{n} u_{n}(x, y, z, t)\right)_{x x x}\right.\right.\right. & \\
& \left.\left.\left.+y^{3}\left(\sum_{n=0}^{\infty} p^{n} u_{n}(x, y, z, t)\right)_{y y y}+z^{3}\left(\sum_{n=0}^{\infty} p^{n} u_{n}(x, y, z, t)\right)_{z z z}\right]\right]\right)
\end{aligned}
$$




$$
\begin{aligned}
& p^{0} u_{0}(x, y, z, t)+p^{1} u_{1}(x, y, z, t)+p^{2} u_{2}(x, y, z, t)+\ldots \\
& =x^{4} y^{4} z^{4} t-p\left(\mathcal { L } ^ { - 1 } \left[\frac { 1 } { 7 2 s } \mathcal { L } \left[x^{3}\left(p^{0} u_{0}(x, y, z, t)+p^{1} u_{1}(x, y, z, t)+\ldots\right)_{x x x}\right.\right.\right. \\
& +y^{3}\left(\sum_{n=0}^{\infty} p^{0} u_{0}(x, y, z, t)+p^{1} u_{1}(x, y, z, t)+\ldots\right)_{y y y} \\
& \left.\left.+z^{3}\left(p^{0} u_{0}(x, y, z, t)+p^{1} u_{1}(x, y, z, t)+\ldots\right)_{z z z}\right]\right)
\end{aligned}
$$

Comparing the coefficient of like power of $p$, the following approximations are obtained;

$$
\begin{aligned}
p^{0}: u_{0}(x, y, z, t) & =x^{4} y^{4} z^{4} t \\
p^{1}: u_{1}(x, y, z, t) & =-\mathcal{L}^{-1}\left[\frac{1}{72 s} \mathcal{L}\left[x^{3} u_{0 x x x}+y^{3} u_{0 y y y}+z^{3} u_{0 z z z}\right]\right] \\
& =-\mathcal{L}^{-1}\left[\frac{1}{72 s} \mathcal{L}\left[x^{3}\left(x^{4} y^{4} z^{4} t\right)_{x x x}+y^{3}\left(x^{4} y^{4} z^{4} t\right)_{y y y}+z^{3}\left(x^{4} y^{4} z^{4} t\right)_{z z z}\right]\right] \\
& =-x^{4} y^{4} z^{4} \frac{t^{2}}{2 !} \\
p^{2}: u_{2}(x, y, z, t) & =-\mathcal{L}^{-1}\left[\frac{1}{72 s} \mathcal{L}\left[x^{3} u_{1 x x x}+y^{3} u_{1 y y y}+z^{3} u_{1 z z z}\right]\right] \\
& =-\mathcal{L}^{-1}\left[\frac{1}{72 s} \mathcal{L}\left[x^{3}\left(-x^{4} y^{4} z^{4} \frac{t^{2}}{2 !}\right)_{x x x}+y^{3}\left(-x^{4} y^{4} z^{4} \frac{t^{2}}{2 !}\right)_{y y y}+z^{3}\left(-x^{4} y^{4} z^{4} \frac{t^{2}}{2 !}\right)_{z z z}\right]\right] \\
& =x^{4} y^{4} z^{4} \frac{t^{3}}{3 !}
\end{aligned}
$$

Therefore the solution $u(x, t)$ is given by :

$$
\begin{aligned}
u(x, y, z, t) & =u_{0}(x, y, z, t)+u_{1}(x, y, z, t)+u_{2}(x, y, z, t)+u_{3}(x, y, z, t)+\ldots \\
& =x^{4} y^{4} z^{4} t-x^{4} y^{4} z^{4} \frac{t^{2}}{2 !}+x^{4} y^{4} z^{4} \frac{t^{3}}{3 !}-x^{4} y^{4} z^{4} \frac{t^{4}}{4 !}+\ldots \\
& =x^{4} y^{4} z^{4}\left(t-\frac{t^{2}}{2 !}+\frac{t^{3}}{3 !}-\frac{t^{4}}{4 !}+\ldots\right) \\
& =x^{4} y^{4} z^{4}\left(1-e^{-t}\right)
\end{aligned}
$$

Example 4 Consider the following nonlinear KDV equation.

$$
v_{t}-a v v_{x}+v_{x x x}=0, a \in R-\{0\}
$$

with the initial condition $v(x, 0)=\frac{1}{a}(x-1)$.

Taking the Laplace transform on both sides of Eq. (3.13), we get

$$
\begin{array}{r}
\mathcal{L}\left[v_{t}\right]-a \mathcal{L}\left[v v_{x}\right]+\mathcal{L}\left[v_{x x x}\right]=\mathcal{L}[0] \\
s v(x, s)-u(x, 0)-a \mathcal{L}\left[v v_{x}\right]+\mathcal{L}\left[v_{x x x}\right]=0 \\
s \mathcal{L}[v(x, t)]-\frac{1}{a}(x-1)-a \mathcal{L}\left[v v_{x}\right]+\mathcal{L}\left[v_{x x x}\right]=0 \\
\mathcal{L}[v(x, t)]=\frac{1}{a s}(x-1)+\frac{a}{s} \mathcal{L}\left[v v_{x}\right]-\frac{1}{s} \mathcal{L}\left[v_{x x x}\right]
\end{array}
$$

The inverse of Laplace transform implies that

$$
\begin{aligned}
v(x, t) & =\mathcal{L}^{-1}\left[\frac{1}{a s}(x-1)\right]+\mathcal{L}^{-1}\left[\frac{a}{s} \mathcal{L}\left[v v_{x}\right]\right]-\mathcal{L}\left[\frac{1}{s} \mathcal{L}\left[v_{x x x}\right]\right] \\
& =\frac{1}{a}(x-1)+6 \mathcal{L}^{-1}\left[\frac{1}{s} \mathcal{L}\left[v v_{x}\right]\right]-\mathcal{L}^{-1}\left[\frac{1}{s} \mathcal{L}\left[v_{x x x}\right]\right]
\end{aligned}
$$


Now,we apply the homotopy perturbation method, we get

$$
\begin{gathered}
v(x, t)=\frac{1}{a}(x-1)+p \mathcal{L}^{-1}\left[\frac{a}{s} \mathcal{L}\left[v v_{x}\right]-\frac{1}{s} \mathcal{L}\left[v_{x x x}\right]\right] \\
\sum_{n=0}^{\infty} p^{n} v_{n}(x, t)=\frac{1}{a}(x-1)+p \mathcal{L}^{-1}\left[\frac{a}{s} \mathcal{L}\left[\sum_{n=0}^{\infty} p^{n} H_{n}(u)\right]-\frac{1}{s} \mathcal{L}\left[\left(\sum_{n=0}^{\infty} p^{n} v_{n}(x, t)\right)_{x x x}\right]\right] \\
p^{0} v_{0}(x, t)+p^{1} v_{1}(x, t)+p^{2} v_{2}(x, t)+\ldots= \\
-\frac{1}{a}(x-1)+p \mathcal{L}^{-1}\left[\frac{a}{s} \mathcal{L}\left[p^{0} H_{0}(u)+p^{1} H_{1}(u)+\ldots\right]\right. \\
\left.-\frac{1}{s} \mathcal{L}\left[\left(p^{0} v_{0}(x, t)+p^{1} v_{1}(x, t)+\ldots\right)_{x x x}\right]\right]
\end{gathered}
$$

Comparing the coefficient of like power of $p$, the following approximations are obtained;

$$
\begin{aligned}
p^{0}: v_{0}(x, t) & =\frac{1}{a}(x-1) \\
p^{1}: v_{1}(x, t) & =\mathcal{L}^{-1}\left[\frac{a}{s} \mathcal{L}\left[H_{0}(u)\right]-\frac{1}{s} \mathcal{L}\left[v_{0 x x x}\right]\right] \\
& =\mathcal{L}^{-1}\left[\frac{a}{s} \mathcal{L}\left[v_{0} v_{0 x}\right]-\frac{1}{s} \mathcal{L}\left[v_{0 x x x}\right]\right] \\
& =\mathcal{L}^{-1}\left[\frac{a}{s} \mathcal{L}\left[\left(\frac{1}{a}(x-1)\right)\left(\frac{1}{a}(x-1)\right)_{x}\right]-\frac{1}{s} \mathcal{L}\left[\left(\frac{1}{a}(x-1)\right)_{x x x}\right]\right] \\
& =\frac{1}{a}(x-1) t \\
p^{2}: v_{2}(x, t) & =\mathcal{L}^{-1}\left[\frac{a}{s} \mathcal{L}\left[H_{1}(u)\right]-\frac{1}{s} \mathcal{L}\left[v_{1 x x x}\right]\right] \\
& =\mathcal{L}^{-1}\left[\frac{a}{s} \mathcal{L}\left[v_{0} v_{1 x}+v_{1} v_{0 x}\right]-\frac{1}{s} \mathcal{L}\left[v_{1 x x x}\right]\right] \\
& =\mathcal{L}^{-1}\left[\frac{1}{s} \mathcal{L}\left[\left(\frac{1}{a}(x-1)\right)\left(\frac{1}{a} t\right)+\left(\frac{1}{a}(x-1)(t)\left(\frac{1}{a}\right)\right)\right]-\frac{1}{s} \mathcal{L}[0]\right] \\
& =\frac{1}{a}(x-1) t^{2} \\
& \vdots \\
v(x, t)= & v_{0}(x, t)+v_{1}(x, t)+v_{2}(x, t)+\ldots \\
= & \frac{1}{a}(x-1)+\frac{1}{a}(x-1) t+\frac{1}{a}(x-1) t^{2}+\frac{1}{36}(x-1) t+\ldots \\
= & \frac{x-1}{a(1-t)}
\end{aligned}
$$

\section{Acknowledgement}

The authors would like to thank The Thailand Research Fund and Office of the Higher Education Commission and Maejo University, Chiang Mai, Thailand for financial support and also Prof.Amnuay Kananthai Department of Mathematics, Chiang Mai University for the helpful of discussion.

\section{References}

He, J. H. (1990). Homotopy pertubation technique, Computer Methods in Applied Mechanics and Engineering, 257-262. https://doi.org/10.1016/S0045-7825(99)00018-3

He, J. H. (2006). Homotopy pertubation method for solving boundary value problem,Physics Letter, 350(1-2), 87-88. https://doi.org/10.1016/j.physleta.2005.10.005 
Kumar, D., \& Singh, J. (2013). An application of Homotopy Pertubation Transform Method to Fractional Heat and Wave-Like Equations, Journal os Fractional Calculus and Applications, 4(2), 290-302.

Momani, S., \& Odibat, Z. (2007). Comparison between the homotopy pertubation method and the variational iteration method for linear fractional partial differential equations, Computation Math Application., 910-918. https://doi.org/10.1016/j.camwa.2006.12.037

Momani, S., \& Odibat, Z. (2007). Homotopy pertubation method for nonlinear partial differential equations of fractional order, Physics Letter, A 365(56), 345-350. https://doi.org/10.1016/j.physleta.2007.01.046

Momani, S. (2005). Analytic approximation solution for fractional heat-like and wave-like equations with variable coefficients using the decomposition method, Apply Math Computation, 459-472.

https://doi.org/10.1016/j.amc.2004.06.025

\section{Copyrights}

Copyright for this article is retained by the author(s), with first publication rights granted to the journal.

This is an open-access article distributed under the terms and conditions of the Creative Commons Attribution license (http://creativecommons.org/licenses/by/4.0/). 